\title{
Das Netzwerk Europäische Bewegung Deutschland
}

\author{
Katharina Wolf \\ Mitglied der Kommission Öffentliches Recht, Europa- und Völ- \\ kerrecht des djb; Regierungsdirektorin, Berlin
}

Der djb ist seit zwei Jahren Mitglied im Netzwerk Europäische Bewegung Deutschland (EBD). Im Juni ist nun das djb-Mitglied Katharina Wolf in den Vorstand gewählt worden. Daher soll hier kurz das Netzwerk EBD vorgestellt werden.

Das Netzwerk Europäische Bewegung Deutschland (EBD $)^{1}$ ist der Zusammenschluss von 201 Interessengruppen aus nahezu allen gesellschaftlichen Bereichen: Wirtschafts- und Berufsverbände, Gewerkschaften, Bildungsträger, wissenschaftliche Institute, Stiftungen, Parteien und Unternehmen. Seit über 60 Jahren informiert das Netzwerk EBD über Europa-Politik, es organisiert und fördert den europapolitischen Dialog zwischen Gesellschaft und Politik in Deutschland.

Nicht nur durch die zunehmende Europäisierung des deutschen Rechts sondern gerade vor dem Hintergrund des Inkrafttretens des Vertrags von Lissabon findet Europa nun auch deutlicher in den einzelnen Mitgliedstaaten selbst statt. Hinzu kommt, dass mit Artikel 11 Absatz 1 und Absatz 2 EUV die Zivilgesellschaft ausdrücklich im Vertrag von Lissabon genannt ist. Darin werden die europäischen Organe verpflichtet, den Bürgerinnen und Bürgern sowie den repräsentativen Verbänden die Möglichkeit zu geben, sich öffentlich in europäischen Angelegenheiten zu äußern und sich mit ihr auszutauschen. Es soll ein offener, transparenter und regelmäßiger Dialog zwischen Zivilgesellschaft, repräsentativen Verbänden einerseits und den Organen der EU andererseits gepflegt werden. Das Netzwerk EBD greift mit seinem Motto „Europapolitik wirkt nach innen - Europapolitik ist deutsche Politik“ diesen Gedanken auf.

Wie sieht die Arbeit des EBD praktisch aus? Die europapolitischen Expertisen, Informationen und Aktionen der Mitgliedsorganisationen werden im Netzwerk EBD gebündelt, vernetzt und verstärkt. Zusätzlich werden politisch aktuell Arbeitsgruppen, strategische Partnerschaften und Dialog-Plattformen geschaffen, in denen Politik und organisierte Zivilgesellschaft an einem Tisch sitzen. Das Netzwerk EBD arbeitet eng mit den Re- präsentanzen der Europäischen Kommission (KOM) und des Europäischen Parlamentes (EP) zusammen. In der Reihe „Europäische Vorausschau“ wurde zum Beispiel das „Stockholmer Programm“, der Aktionsplan der EU für Justiz und Inneres für die nächsten zehn Jahre, vorgestellt. Auch die jeweiligen Ratspräsidentschaften stellen sich den deutschen Interessengruppen mit ihrem jeweiligen Programm in dieser Veranstaltungsreihe vor. Die Bundesregierung unterrichtet die Netzwerkmitglieder in der Reihe „EU De-Briefing“ unmittelbar im Anschluss an die europäischen Fachministerräte und die Europäischen Räte über Inhalte und Verlauf dieser Treffen. Beim „EU-Stammtisch Brüssel in Berlin“ treffen sich ehemalige Brüsseler Interessenvertreter(innen) zum informellen Erfahrungsaustausch. Die Mitgliedsorganisationen haben die Möglichkeit, sich im Internetangebot des Netzwerks EBD unter „Europa-News“ mit den eigenen europapolitischen Profilen am Dialog, beispielsweise bei Konsultationen zu Grünbüchern oder im Vorfeld von Legislativakten, zu beteiligen. Aus djb-Sicht ebenfalls interessante Aktivitäten und Angebote sind: Expert(inn)en-Datenbanken, Preis Frauen Europas, Förderung internationaler Bildungsprojekte, Europäischer Schüler(innen)wettbewerb, wissenschaftliche Kooperationsprojekte mit Unternehmen, Instituten und Stiftungen.

Das Netzwerk EBD ist zudem auch regional in den Bundesländern präsent. Außerdem ist die Europäische Bewegung in 43 Ländern Europas vertreten.

Der djb engagiert sich schon lange auch europapolitisch. Die 1994 eingesetzte Kommission Europarecht und Internationales Recht des djb hat die Idee einer europäischen Juristinnenvereinigung entwickelt. Daraus ist EWLA (European Women Lawyers Association) entstanden, die nunmehr bereits seit zehn Jahren existiert. Denn es hatte sich als sinnvoll und notwendig herausgestellt, auf europäischer Ebene durch eine europaweite Organisation von und für Juristinnen vertreten zu sein.

Mit der Mitgliedschaft im Netzwerk EBD kann sich der djb auch in Deutschland und damit vor Ort europapolitisch vernetzen.

1 〈http://www.europaeische-bewegung.de/> (Zugriff 14.10.2010).

\section{Der djb gratuliert}

\section{Renate Damm}

zum 75. Geburtstag. Am 27. September 1935 wurde sie in Wandsbek geboren. Nach dem Studium in Hamburg und Erlangen - parallel zu einer Schauspielausbildung - trat sie als Syndikus-Anwältin in die Rechtsabteilung des Axel Springer Verlages ein und war zuletzt Leiterin der Stabsabteilung Recht. 1996 wechselte sie in das Hamburger Anwaltsbüro Redeker
Schön Dahs \& Sellner und gründete 2000 die Sozietät Damm und Mann.

Renate Damm war Lehrbeauftragte im Medienrecht an der Universität München und Dozentin für Presserecht an der Hochschule für Musik und Theater in Hamburg. Sie ist Mitglied der Arbeitsgemeinschaft der Verlagsjustitiare sowie des Studienkreises für Presserecht und Pressefreiheit. Hier, wie 


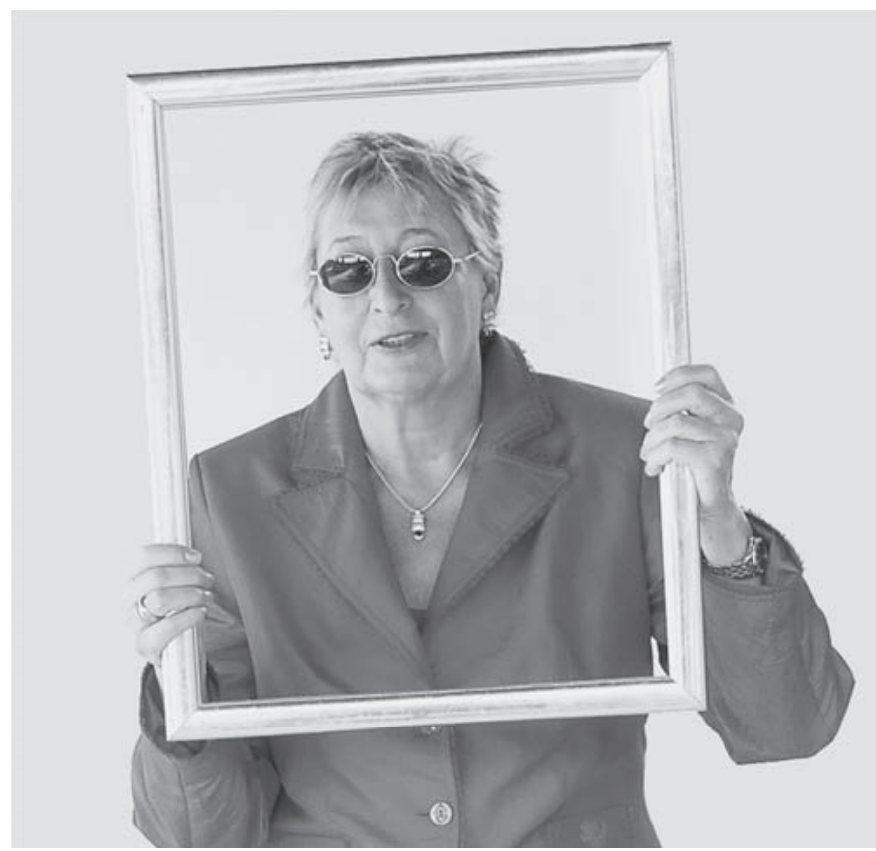

$\Delta$ Rechtsanwältin Renate Damm, Foto: Hans Ilgmoser.

auch in anderen Gremien, war sie die erste und lange Zeit die einzige Frau. Dazu gehörte zum damaligen Zeitpunkt Mut und Durchsetzungskraft, an der es ihr nie fehlte.

Seit 1973 ist sie aktives Mitglied des djb. Nach einigen Jahren Arbeit im Bundesvorstand war sie von 1983 bis 19891. Vorsitzende. Seit 2008 ist sie Ehrenpräsidentin des djb.

Renate Damm betont immer die Notwendigkeit von Netzwerken und ist selbst aktive Netzwerkerin - innerhalb ihres Unternehmens, extern in vielen Gremien, aber auch für den djb. Sie begeistert stets viele neue Mitglieder für den djb, betreibt Mentoring und fördert viele junge Kolleginnen, zum Teil hat sie sie selbst ausgebildet für ihre Arbeit in den Verlagen. 1990 erhielt sie das Bundesverdienstkreuz. Für ihre „besonderen Verdienste um die Verwirklichung der Gleichberechtigung und Gleichstellung der Frauen in Gesellschaft, Beruf und Familie“ überreichte der damalige Bundespräsident Richard von Weizsäcker ihr 1990 das Bundesverdienstkreuz Erster Klasse.

Renate Damm hat ihre beruflichen Ziele - scheinbar leicht erreicht. Sie verlor dabei aber nie aus den Augen, dass nicht alle Frauen die gleichen Karrierechancen hatten und haben wie sie. Sie ist, wie sie sich selbst bezeichnet, eine „glühende Anhängerin der Frauenquote" und der Meinung, dass ohne die Quote nicht viel bewegt worden wäre und noch viel bewegt werden muss - z.B. in der Wirtschaft. Sie ist als „role model“ für uns unverzichtbar - für alle Generationen von Juristinnen. Jüngeren Frauen hat sie als Mutter eines Sohnes gezeigt, dass Beruf und Familie miteinander vereinbar sind und es möglich ist, auch in der Wirtschaft die Leiter bis ganz nach oben zu erklimmen. Kolleginnen mit langjähriger Berufserfahrung dient sie als Vorbild, auch nach vielen Berufsjahren eine neue Herausforderung zu suchen. Hierbei ist sie selbst sehr erfolgreich.

Renate Damm zeigt uns, dass bei aller beruflichen und ehrenamtlichen Tätigkeit auch andere Interessen ihren Platz haben und wichtig sind, um Abstand zu haben. Sie läuft Ski und malt. Vor allen Dingen ist sie aber seit Studienzeiten dem Theater treu geblieben - auch ihre Begeisterung dafür kann sie mitreißend vermitteln.

\section{Gabriele C. Klug}

zur Wahl als Beigeordnete und Kämmerin durch den Rat der Stadt Köln am 7. Oktober 2010. Sie soll ihr Amt am 8. Dezember 2010 antreten. Gabriele C. Klug, geb. 1955 in Frankfurt am Main, ist Juristin mit fachlichen Schwerpunkten im Kollektivarbeitsrecht und im Verfassungsrecht. Nach verschiedenen Studienaufenthalten im Ausland, u.a. an der London School of Economics und ihrem Zweiten Staatesexamen war sie von 1983 bis 1986 als Rechtsanwältin in Frankfurt am Main tätig.

Nach Stationen in der Hessischen Staatskanzlei und im Landtag (ab 1992 als jüngste Leitende Ministerialrätin der Hessischen Landesverwaltung) war sie von 1994 bis 2000 hauptamtliche Bürgermeisterin der Stadt Rüsselsheim, Mitglied im Präsidium des Hessischen Städtetags und des Hauptausschusses des Deutschen Städtetags. Sie war Aufsichtsratsmitglied in einem Unternehmen der Wohnungswirtschaft und Mitglied der Krankenhauskommission. Von 2000 bis 2005 war sie wiederum als Rechtsanwältin tätig. Außerdem war sie stellvertretendes Mitglied des Staatsgerichtshofs des Landes Hessen. Seit 2005 ist sie Beigeordnete und Kämmerin der Stadt Wesel, Mitglied des Präsidiums des Städte- und Gemeindebundes NRW, Mitglied im Verwaltungsrat der Gemeindeprüfungsanstalt (stellv.) und des Hauptausschusses des Deutschen StGB (stellv.), sie ist Mitglied im Aufsichtsrat der örtlichen Stadtwerke und Mitglied im Aufsichtsrat der regionalen Hafenentwicklungsgesellschaft.

Seit 1987 ist sie Mitglied im djb und engagiert sich seit 2005 in der Kommission Arbeits-, Gleichstellungs- und Wirtschaftsrecht. Ihr Arbeitsschwerpunkt liegt im Bereich Corporate und Public Corporate Governance.

Sie ist Mitglied des Vorstands von Transparency International Deutschland e.V. (seit 2007) - verantwortlich u.a. für den Bereich Vergabewesen und Korruptionsregister. Gabriele C. Klug ist verheiratet, hat eine erwachsene Tochter und ist seit 1992 Mitglied der Grünen.

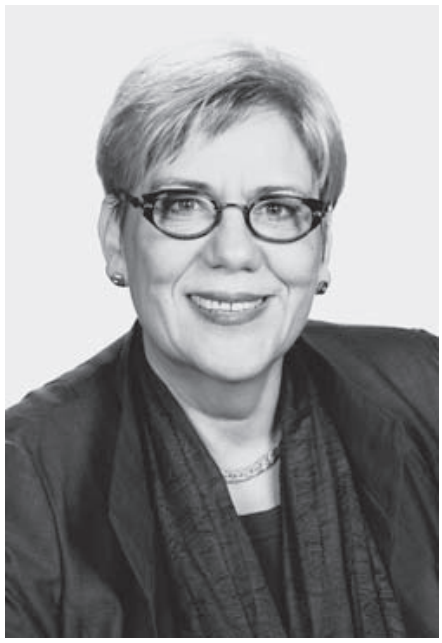

$\checkmark$ Gabriele C. Klug, Beigeordnete, Leitende Ministerialrätin a. D. 


\section{Ingrid Weber}

zum 70. Geburtstag. Ingrid Weber ist Vorsitzende Richterin am Landesarbeitsgericht Berlin i.R. und leidenschaftliche Arbeitsrechtlerin auch über ihre berufliche Tätigkeit hinaus. Für die Gleichstellung der Geschlechter und diskriminierungsfreie Arbeits- und Lebensbedingungen hat sie viel erreicht.

Ein besonderes Anliegen sind ihr die Gleichstellungsgesetze allen voran in Berlin. Das Berliner Frauenfördergesetz (später umbenannt in LGG Berlin) trat vor 15 Jahren in Kraft. Die damals zuständige Senatorin Anne Klein hatte im Vorfeld eine Expertinnenrunde einberufen, deren Mitglied Ingrid Weber war. Berlin hatte mit der Schaffung des Gesetzes eine Vorreiterrolle, die Widerstände waren beträchtlich. Ingrid Weber moderierte viele Veranstaltungen zum LGG, um für die gesetzlichen Regelungen zu werben. Zehn Jahre später war sie als Sachverständige und Moderatorin ebenfalls beteiligt, als die Rechtsverordnung zu formulieren war, mit der die Koppelung öffentlicher Auftragsvergabe mit Frauenförderung näher ausgestaltet wurde.

Frauenpolitisch ebenfalls sehr wichtig ist ihr die Gleichstellung der Geschlechter in der Privatwirtschaft. Dr. Bergmann berief 1999 als zuständige Bundesministerin eine Expertenrunde mit Vertretern und Vertreterinnen aus Wissenschaft und Praxis, Unternehmen und Gewerkschaften ein, der Ingrid Weber als frauenpolitisch engagierte Richterin angehörte. Es ging darum, Rahmenbedingungen in Hinblick auf die Akzeptanz eines entsprechenden Gesetzes abzuklären. 2000 beauftragte die Ministerin eine sechsköpfige Expertinnengruppe unter Führung von Prof. Dr. Heide Pfarr mit der Formulierung eines Gesetzentwurfs, der 2001 mit ihrer tatkräftigen Unterstützung vorgelegt wurde und auch heute noch Ausgangspunkt für die Diskussion um ein Gesetz zur Gleichstellung der Geschlechter in der Privatwirtschaft ist.

Ingrid Weber engagiert sich seit 1986 im Deutschen Juristinnenbund und war in mehreren Kommissionen und Arbeitsstäben Mitglied (Arbeitsrecht und moderne Technologien, Gleichstellungskommission, Arbeits- und Sozialrecht, Deutsch-deutsche Rechtsbeziehungen). Von 2001 bis 2005 leitete sie die Kommission Arbeits-, Gleichstellungs- und Wirtschaftsrecht, war in dieser Funktion auch Mitglied des Bundesvorstandes und verantwortlich für zahlreiche wichtige Stellungnahmen des djb. Alle Stellungnahmen entstanden in echter Teamarbeit, Ingrid Weber leitete die Kommission unprätentiös und förderte die besonderen Stärken jedes einzelnen Kommissionsmitgliedes. Nicht nur innerhalb der Kommission sondern auch im Vorstand hatte sie hohe Integrationskraft und dies auch bei kniffligen Themen und manchmal schwierigen Abstimmungsprozessen.

Ingrid Weber gehört zu den Frauen im djb, die andere anspornen und begeistern können. Sie nimmt auch und gerade jüngere und unbekanntere Mitglieder des djb ernst und integriert sie auf heitere Weise in die gemeinsame Arbeit. Ihre Gastfreundlichkeit ist legendär und ihr Rat nach wie vor willkommen. Ich persönlich habe unglaublich viel von ihr gelernt. (Christine Fuchsloch)

\section{Women Power in Brussels - how and why do women lobby differently?}

\section{Podiumsdiskussion am 20. September 2010 in der Nordrhein-Westfälischen Landesvertretung in Brüssel}

\section{Dr. Claudia Schöler}

Mitglied im Vorstand der Regionalgruppe Brüssel des djb; VDMA European Office, Brüssel

„Women Power in Brussels - how and why do women lobby differently? “ - unter diesem Motto lud die Regionalgruppe Brüssel im djb zusammen mit dem niederländischen Frauennetzwerk „Brussels NV“ am 20. September 2010 zu einer Podiumsdiskussion in die Nordrhein-Westfälische Landesvertretung in der EU-Hauptstadt. Zwei prominente Brüsseler Interessenvertreterinnen gaben Einblick in ihre politische Arbeit: Constanze Picking, Direktorin der EU-Unternehmensrepräsentanz von Daimler, und Truus Huisman, Leiterin des Public Affairs-Büros von Unilever in Brüssel. Carla Joosten, Korrespondentin des niederländischen Politik-Magazins „Elsevier“ führte durch die Diskussion.
Gesprächsstoff gab es reichlich - angesichts der hohen Sprosse, die beide Damen auf der Karriereleiter erklommen haben, lagen Fragen zum persönlichen beruflichen Werdegang und der Vereinbarkeit von Beruf und Familie genauso auf der Hand wie Fragen zur Kunst der politischen Interessenvertretung in der EU-Hauptstadt, in der sich über 15.000 Lobbyisten tummeln. Wie haben es beide Frauen in der „Männerwelt“ ihrer Unternehmen „nach oben“ geschafft? Brauchen wir die Frauenquote und gibt es die viel gescholtene „gläserne Decke“ (letztere, das wurde schnell klar, war eine rhetorische Frage!)? Wie hat sich die politische Interessenvertretung im Laufe der Jahre verändert, welche Rolle spielt die Transparenzinitiative der EU-Institutionen und inwieweit kann die Industrie von Kampagnen der Nichtregierungsorganisationen lernen? Auch die Frage nach Erfolgsgeschichten und weniger erfolgreichem Lobbying war erlaubt. Offene Worte, interessante Einblicke 